\title{
Model-Free Precompensator and Feedforward Tuning Based on the Correlation Approach
}

\author{
Alireza Karimi, Mark Butcher and Roland Longchamp
}

\begin{abstract}
High performance output tracking can be achieved by precompensator or feedforward controllers based on the inverse of the closed-loop system or the plant model. However, it has been shown that these inverse controllers can affect adversely the tracking performance in the presence of model uncertainty. In this paper, a model-free approach based on only one set of acquired data from a simple closed-loop experiment is used to tune the controller parameters. The approach is based on the decorrelation of the tracking error and the desired output and is not asymptotically sensitive to noise and disturbances. By a frequency-domain analysis of the criterion, it is shown that the weighted two-norm of the difference between the controller and the inverse of the plant model (or the closed-loop transfer function) can be minimized. The method is successfully applied to a high precision position control system.
\end{abstract}

\section{INTRODUCTION}

Two-degree of freedom controllers are largely used when disturbance rejection and reference signal tracking are both considered as closed loop performance criteria. In many cases, the feedback controller is first designed to ensure the robust stability and satisfy the disturbance rejection specification. Then, in the second step, a precompensator (Fig. 1) or a feedforward controller (Fig. 2) is designed to improve the tracking performance. If the plant model is perfectly known, this problem can be converted to a standard model matching problem and can be solved analytically or using the convex optimization algorithms. However, a perfect model of the plant is never available and a nominal model with some uncertainty bounds should be considered for the design [1].

Another approach, when a mathematical model of the plant is not available, is to tune directly the controller parameters using the data acquired from some simple experiments. Using the measured data, the controller parameters are tuned to minimize a control criterion which is normally the twonorm of a performance error. Iterative Feedback Tuning (IFT) is a model-free approach for tuning the parameters of two-degree of freedom controllers based on some specific closed-loop experiments to compute an unbiased estimate of the gradient of the control criterion [2]. Separate tuning of the feedforward and the feedback controllers is proposed to improve the tracking performance using the IFT approach in [3]. Iterative Learning Control (ILC) is also used for feedforward control of systems with periodic reference or

\footnotetext{
The authors are with the Automatic Control Laboratory of Ecole Polytechnique Fédéral de Lausanne (EPFL), 1015 Lausanne, Switzerland.

Corresponding author: alireza.karimi@epfl.ch

This work is supported by Office Fédéral de la Formation Professionnelle et de la Technologie under the CTI-projet no. 7049.1 IWS-IW
}

disturbance signal. A characteristic feature of data-driven approaches is the sensitivity of the controller parameters (in IFT) or the control input (in ILC) to stochastic disturbances. Moreover, these methods require many experiments on the system. The main interest of a new controller tuning method called Virtual Reference Feedback Tuning (VRFT) is that only one set of data is required to tune a controller for the model reference problem [4]. However, the drawback is that an approximation of the control criterion is minimized.

Recently, a new data-driven iterative method based on the correlation approach has been proposed and successfully applied to a magnetic suspension system [5] as well as a benchmark problem [6]. An overview of this approach together with the theoretical results can be found in [7]. The main idea is instead of minimizing the performance error, to make it uncorrelated with the reference signal. It can be shown in this case that the noise has asymptotically no effect on the controller parameter estimates.

In this paper, the correlation approach will be used to tune the parameters of the precompensator or feedforward controller such that the tracking error becomes uncorrelated with the desired output. In contrast to the feedback controller tuning, only one set of data from a simple closedloop experiment is used to tune the parameters of the precompensator or feedforward controller. Moreover, with an appropriate parameterization, not only the stability of the tuned controller is guaranteed but also the minimization of the control criterion becomes a classical least squares problem. The optimal parameters are derived for the precompensator and feedforward controller. Frequency-domain analysis of the criterion shows that the proposed approach gives asymptotically an optimal model-free solution to the model matching problem in two norm.

The paper is organized as follows. Notation and preliminaries about the correlation approach are given in Section II. Precompensator tuning scheme together with the one shot tuning algorithm and frequency-domain analysis are presented in Section III. Section IV studies the tuning of the feedforward controllers. Simulation results and experimental results for high precision position control of a linear synchronous motor are presented in Section V. Finally, the concluding remarks are given in Section VI.

\section{PRELIMINARIES}

Let the output $y(t)$ of a SISO linear time-invariant plant model $P\left(q^{-1}\right)$ be described by:

$$
y(t)=P\left(q^{-1}\right) u(t)+v(t)
$$




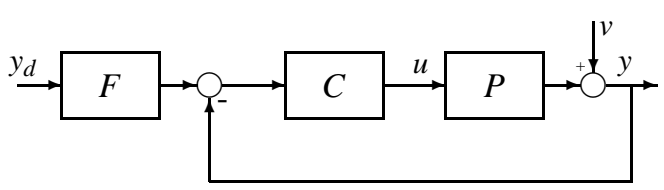

Fig. 1. Closed-loop system with precompensator

where $u(t)$ is the plant input, $v(t)$ a zero-mean weakly stationary random process and $q^{-1}$ the backward-shift operator. Assume that the controller $C\left(q^{-1}\right)$ stabilizes the plant model $P\left(q^{-1}\right)$ in closed-loop with unit feedback. It is supposed that the plant model $P\left(q^{-1}\right)$ and the controller $C\left(q^{-1}\right)$ are unknown and the objective is to improve the closed-loop tracking error $e(t)=y_{d}(t)-y(t)$ using only one set of data acquired in closed-loop operation. In practice, two different control approaches are used to reduce the tracking error:

- Precompensator: Filtering the desired output by a precompensator before applying it as a reference signal to the closed-loop system (modifying the reference signal),

- Feedforward: Adding the filtered desired output to the controller output (modifying the plant input).

Fig. 1 shows the block diagram of a two-degree of freedom controller with a precompensator. Principally, when $y_{d}$ is a priori known, the precompensator $F$ should be a stable (not necessarily causal) approximation of the inverse of the closed loop transfer function $T=C P(1+C P)^{-1}$. For the feedforward scheme (Fig. 2), however, the feedforward controller $F$ should be a stable approximation of the inverse of the plant model $P$.

\section{A. Controller Parameterization}

Let $F$ be parameterized as

$$
F\left(\rho, q^{-1}\right)=\beta^{T}\left(q^{-1}\right) \rho
$$

where $\rho^{T}=\left[\rho_{0}, \rho_{1}, \ldots, \rho_{n_{\rho}}\right]$ is the vector of controller parameters and $\beta\left(q^{-1}\right)$ the vector of linear discrete-time transfer operators

$$
\beta^{T}\left(q^{-1}\right)=\left[\beta_{0}\left(q^{-1}\right), \beta_{1}\left(q^{-1}\right), \ldots, \beta_{n_{\rho}}\left(q^{-1}\right)\right] .
$$

In the sequel, for simplicity, we suppose that $\beta^{T}\left(q^{-1}\right)=$ $\left[q^{\delta}, q^{\delta-1}, \ldots, q^{\delta-n_{\rho}}\right]$ which leads to the following FIR model for $F$ :

$$
F\left(\rho, q^{-1}\right)=\rho_{0} q^{\delta}+\rho_{1} q^{\delta-1}+\cdots+\rho_{n_{\rho}} q^{\delta-n_{\rho}}
$$

where $\delta$ is a positive scalar. In fact, the desired output is applied $\delta$ sampling periods in advance to the real system to improve the tracking error [8]. For the sake of simplicity, $q^{-1}$ will be omitted when appropriate in the rest of the paper.

\section{B. Correlation Approach}

It is evident that if the exact inverse of the closed-loop system (for the precompensator case) or the exact inverse of the plant model (for the feedforward case) exists the tracking error $e(t)$ will contain only the contribution of the noise. Hence, it is reasonable to adjust the controller $F$ in such

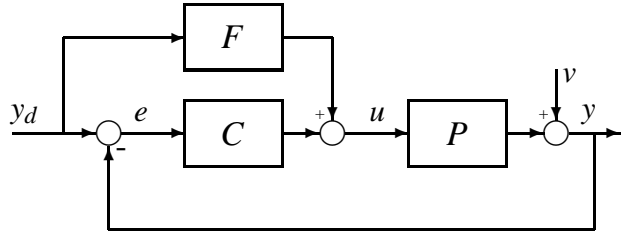

Fig. 2. Closed-loop system with feedforward

a way that the tracking error $e(t)$ be uncorrelated with the desired output. For many systems, the exact inverse does not exist because the system is non minimum phase or of infinite order. As a result, $e(t)$ is always correlated with the desired output. However, it can be considered that a good controller $F$ minimizes the correlation between the tracking error $e(t)$ and the desired output $y_{d}(t)$. In order to formulate this idea as an optimization problem, let the correlation function $f(\rho)$ be defined as:

$$
f(\rho)=E\left\{\left[y_{d}(t)-y(t)\right] \zeta(t)\right\}=E\{e(t) \zeta(t)\}
$$

where $E\{\cdot\}$ denotes the mathematical expectation and

$$
\zeta^{T}(t)=\left[y_{d}(t+n), \ldots, y_{d}(t), y_{d}(t-1), \ldots, y_{d}(t-n)\right]
$$

with $l=2 n+1$ the dimension of $\zeta(t)$ which should be larger than the order of the closed-loop system. In fact $\zeta(t)$ is a vector of instrumental variables correlated with $y_{d}(t)$ and uncorrelated with $v(t)$. Now, a new control criterion based on the correlation approach is defined:

$$
J(\rho)=\|f(\rho)\|_{2}^{2}=f^{T}(\rho) f(\rho)
$$

and the optimal controller parameters are:

$$
\rho^{*}=\arg \min _{\rho} J(\rho) .
$$

Since the control criterion involves the mathematical expectation, an exact solution when only one set of finite number data is available, is not attainable. However, with an ergodicity assumption on the input signals, a good estimate of the correlation function can be given by:

$$
\hat{f}(\rho)=\frac{1}{N} \sum_{t=1}^{N} e(t) \zeta(t)
$$

where $N$ is the number of data and should be large enough with respect to $l$. The estimate of the correlation function leads to the following criterion

$$
J_{N}(\rho)=\|\hat{f}(\rho)\|_{2}^{2}=\hat{f}^{T}(\rho) \hat{f}(\rho) .
$$

The criterion $J_{N}(\rho)$ goes to $J(\rho)$ when $N$ tends to infinity. An optimal minimizer of $J_{N}(\rho)$ can be derived using the least squares algorithm. This solution together with an asymptotic frequency domain analysis is presented in the next sections.

\section{PRECOMPENSATOR TUNING}

In this section, we propose a tuning scheme to find the parameters of the precompensator $F$ based on the correlation approach. 


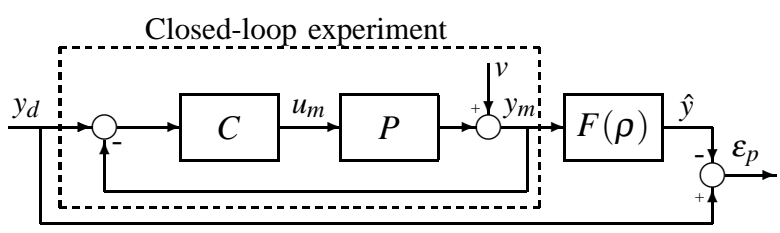

Fig. 3. Precompensator tuning scheme

\section{A. Tuning scheme}

The tracking error can be computed as (see Fig. 1):

$$
e(t)=y_{d}(t)-y(t)=y_{d}(t)-F(\rho) T y_{d}(t)-S v(t)
$$

where $T=C P(1+C P)^{-1}$ and $S=(1+C P)^{-1}$ are the closedloop sensitivity functions. Computing $e(t)$ for different values of $\rho$ requires many experiments on the system that can be avoided by a new tuning scheme in which the place of closed-loop system and precompensator is interchanged so that $F$ acts as a post-compensator (see Fig. 3). It should be mentioned that this can be done only for SISO LTI systems. In this scheme $u_{m}$ and $y_{m}$ are the measured input and output of the plant from a closed-loop experiment with desired output $y_{d}$ as the reference signal. An estimate of the tracking error now can be computed only with one set of data as follows

$$
\begin{aligned}
\varepsilon_{p}(t) & =y_{d}(t)-\hat{y}(t)=y_{d}(t)-F(\rho) y_{m}(t) \\
& =y_{d}(t)-F(\rho) T y_{d}(t)-F(\rho) S v(t)
\end{aligned}
$$

It is clear that in the absence of noise $(v(t) \equiv 0) e(t)$ and $\varepsilon_{p}(t)$ are equal. However, even in the presence of noise we have

$$
f(\rho)=E\{e(t) \zeta(t)\}=E\left\{\varepsilon_{p}(t) \zeta(t)\right\}
$$

if the disturbance signal $v(t)$ is independent of $y_{d}(t)$.

\section{B. Algorithm}

The estimate of the tracking error $\varepsilon_{p}(t)$ can be presented in the linear regression form:

$$
\varepsilon_{p}(t)=y_{d}(t)-F(\rho) y_{m}(t)=y_{d}(t)-\phi^{T}(t) \rho
$$

where

$$
\phi^{T}(t)=\left[y_{m}(t+\delta), y_{m}(t+\delta-1), \ldots, y_{m}\left(t-n_{\rho}+\delta\right)\right] .
$$

This leads to the following expression for the correlation function estimate

$$
\hat{f}(\rho)=\frac{1}{N} \sum_{t=1}^{N} \zeta(t)\left[y_{d}(t)-\phi^{T}(t) \rho\right]=Z-Q \rho
$$

where

$$
Z=\frac{1}{N} \sum_{t=1}^{N} \zeta(t) y_{d}(t) \quad, \quad Q=\frac{1}{N} \sum_{t=1}^{N} \zeta(t) \phi^{T}(t)
$$

Finally, if $Q^{T} Q$ is nonsingular (i.e. $y_{d}$ is sufficiently rich), straightforward calculation gives:

$$
\hat{\rho}=\left(Q^{T} Q\right)^{-1} Q^{T} Z
$$

where $\hat{\rho}$ is the optimal minimizer of the correlation criterion in (10).

\section{Frequency-domain analysis}

The correlation criterion in (7) can be reformulated as

$$
J(\rho)=f^{T}(\rho) f(\rho)=\sum_{\tau=-n}^{n} R_{e y_{d}}^{2}(\tau)
$$

where $R_{e y_{d}}(\tau)$ is the cross-correlation function between the desired output $y_{d}(t)$ and the tracking error $e(t)$ defined by:

$$
\begin{aligned}
R_{e y_{d}}(\tau) & =E\left\{e(t) y_{d}(t-\tau)\right\}=E\left\{\varepsilon_{p}(t) y_{d}(t-\tau)\right\} \\
& =E\left\{\left[y_{d}(t)-F(\rho) T y_{d}(t)\right] y_{d}(t-\tau)\right\}
\end{aligned}
$$

The correlation criterion can be represented in the frequency domain by applying the Parseval's theorem when $n$ tends to infinity:

$$
\begin{aligned}
\lim _{n \rightarrow \infty} J(\rho)=\frac{1}{2 \pi} \int_{-\pi}^{\pi}\left|\Phi_{e_{d}}(\omega)\right|^{2} d \omega \\
\quad=\frac{1}{2 \pi} \int_{-\pi}^{\pi}\left|1-F\left(\rho, e^{-j \omega}\right) T\left(e^{-j \omega}\right)\right|^{2} \Phi_{y_{d}}^{2}(\omega) d \omega
\end{aligned}
$$

where $\Phi_{e y_{d}}(\omega)$ is the cross-spectral density between $e(t)$ and $y_{d}(t)$ and $\Phi_{y_{d}}(\omega)$ is the spectral density of $y_{d}$. This expression shows that:

- The criterion is not asymptotically affected by noise.

- In the ideal case where there exists $\rho^{*}$ such that $F\left(\rho^{*}\right)=$ $T^{-1}$ (i.e. $T$ is minimum phase and $F$ is properly parameterized) $\rho^{*}$ is the minimum of the correlation criterion in (7).

- If $y_{d}(t)$ is white noise the correlation criterion becomes

$$
J(\rho)=\left\|T\left[T^{-1}-F(\rho)\right]\right\|_{2}^{2}
$$

so the difference between $F$ and $T^{-1}$ weighted by the closed-loop transfer function is minimized in the twonorm sense using the correlation approach.

Remark: The model following problem in two-norm also can be treated with this model-free approach. Consider that we aim to compute the precompensator $F$ such that $\|M-F(\rho) T\|_{2}$ be minimized. To proceed, we define $\varepsilon_{M}(t)=$ $M y_{d}(t)-\phi^{T}(t) y_{m}(t)$ and we compute $\rho$ such that $\varepsilon_{M}(t)$ is not correlated with $y_{d}(t)$ which is chosen to be a white noise signal independent of $v(t)$.

\section{Control input weighting}

When a precompensator is added to the feedback controller to improve the tracking performance, it is possible that the control input becomes too large for certain desired outputs. Therefore, it is reasonable to take into account the control input in the design of the precompensator. The measured control input $u_{m}$ that corresponds to the control input when the feedback controller alone is used can be represented by $u_{m}(t)=C S\left[y_{d}(t)-v(t)\right]$ (see Fig. 3). In the presence of the precompensator, an estimate of the control input can be obtained by $u_{f}(t)=F(\rho) u_{m}(t)=\varphi^{T}(t) \rho$ where

$$
\varphi^{T}(t)=\left[u_{m}(t+\delta), u_{m}(t+\delta-1), \ldots, u_{m}\left(t-n_{\rho}+\delta\right)\right]
$$

It is clear that in the absence of noise, $u_{f}(t)$ is equal to $u(t)$ and in the presence of noise we have:

$$
g(\rho)=E\left\{u_{f}(t) \zeta(t)\right\}=E\{u(t) \zeta(t)\} .
$$




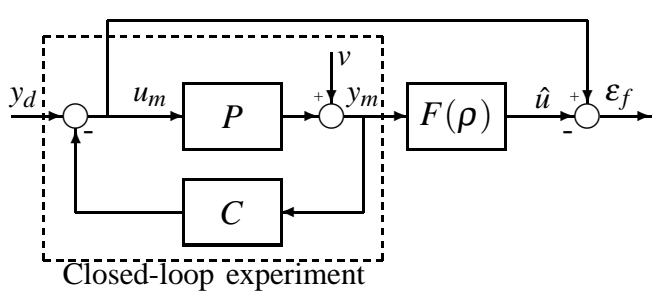

Fig. 4. Feedforward tuning scheme

Now in order to consider the spectrum of the control input in the control design, let the following correlation criterion be defined:

$$
\begin{aligned}
J(\rho) & =f^{T}(\rho) f(\rho)+\lambda g^{T}(\rho) g(\rho) \\
& =\sum_{\tau=-n}^{n} R_{e_{d}}^{2}(\tau)+\lambda R_{u_{f} y_{d}}^{2}(\tau)
\end{aligned}
$$

where $R_{u_{f} y_{d}}(\tau)$ is the cross-spectral density function between the control input estimate and the desired output, and $\lambda$ a positive scalar weighting factor. This new criterion can be interpreted in the frequency-domain as:

$$
\begin{gathered}
\lim _{n \rightarrow \infty} J(\rho)=\frac{1}{2 \pi} \int_{-\pi}^{\pi}\left[\left|\Phi_{e y_{d}}(\omega)\right|^{2}+\lambda\left|\Phi_{u_{f} y_{d}}(\omega)\right|^{2}\right] d \omega \\
=\frac{1}{2 \pi} \int_{-\pi}^{\pi}\left[\left|1-F\left(\rho, e^{-j \omega}\right) T\left(e^{-j \omega}\right)\right|^{2}\right. \\
\left.+\lambda\left|F\left(\rho, e^{-j \omega}\right) C\left(e^{-j \omega}\right) S\left(e^{-j \omega}\right)\right|^{2}\right] \Phi_{y_{d}}^{2}(\omega) d \omega .
\end{gathered}
$$

Therefore, using the criterion in (26) and an appropriate choice of $\lambda$ the magnitude of the frequency response of the control input can be reduced in the frequency range where the spectrum of the desired output is large.

For a finite number of data, an approximation of the criterion can be obtained by

$$
J_{N}(\rho)=\hat{f}^{T}(\rho) \hat{f}(\rho)+\lambda \hat{g}^{T}(\rho) \hat{g}(\rho)
$$

where

$$
\hat{g}(\rho)=\frac{1}{N} \sum_{t=1}^{N} \zeta(t) \varphi^{T}(t) \rho=Q_{u} \rho
$$

and

$$
Q_{u}=\frac{1}{N} \sum_{t=1}^{N} \zeta(t) \varphi^{T}(t)
$$

The global minimum of this criterion is given by:

$$
\hat{\rho}=\left(Q^{T} Q+\lambda Q_{u}^{T} Q_{u}\right)^{-1} Q^{T} Z .
$$

\section{FEEDFORWARD TUNING}

The tuning of the feedforward controller to reduce the tracking error is very similar to that of the precompensator. The main idea is that $F(\rho)$ should make the tracking error $e(t)$ uncorrelated with the desired output $y_{d}(t)$. The tracking error in the feedforward scheme is (see Fig. 2)

$$
e(t)=S y_{d}(t)-F(\rho) P S y_{d}(t)-S v(t) .
$$

Since this signal is not available for every value of $\rho$, we propose a tuning scheme based on only one closed-loop experiment. This scheme is depicted in Fig. 4 and is based on a closed-loop experiment where the desired output $y_{d}$ is added to the control input and the closed-loop reference signal is fixed to zero. The plant input $u_{m}$ and the plant output $y_{m}$ are measured. The measured output filtered by $F(\rho)$ is denoted by $\hat{u}_{m}$ because, in the absence of noise, if $F(\rho) \approx P^{-1}$ it will be an estimate of $u_{m}$. Then, the estimate of the tracking error $\varepsilon_{f}(t)=u_{m}(t)-\hat{u}_{m}(t)$ can be computed by

$$
\varepsilon_{f}(t)=S y_{d}(t)-C S v(t)-F(\rho) P S y_{d}(t)-F(\rho) S v(t) .
$$

It is clear from the above equations that $e(t)$ is equal to $\varepsilon_{f}(t)$ in the absence of disturbance $v(t)$. Moreover, if the disturbance $v(t)$ is independent of $y_{d}(t)$ we have:

$$
f(\rho)=E\{e(t) \zeta(t)\}=E\left\{\varepsilon_{f}(t) \zeta(t)\right\} .
$$

This leads to the following expression in regression form for the correlation function estimate

$$
\hat{f}(\rho)=\frac{1}{N} \sum_{t=1}^{N} \zeta(t)\left[u_{m}(t)-\phi^{T}(t) \rho\right]=Z_{f}-Q \rho
$$

where $\phi^{T}(t)$ and $Q$ are the same as those defined respectively in (16) and (18) for the precompensator tuning and

$$
Z_{f}=\frac{1}{N} \sum_{t=1}^{N} \zeta(t) u_{m}(t)
$$

Therefore the parameters of the optimal controller are

$$
\hat{\rho}=\left(Q^{T} Q\right)^{-1} Q^{T} Z_{f}
$$

A similar frequency-domain analysis using the Parseval's relation gives the following expression for the correlation criterion

$$
\begin{aligned}
& \lim _{n \rightarrow \infty} J(\rho)=\frac{1}{2 \pi} \int_{-\pi}^{\pi}\left|\Phi_{e y_{d}}(\omega)\right|^{2} d \omega= \\
& \frac{1}{2 \pi} \int_{-\pi}^{\pi}\left|S\left(e^{-j \omega}\right)\right|^{2}\left|1-F\left(\rho, e^{-j \omega}\right) P\left(e^{-j \omega}\right)\right|^{2} \Phi_{y_{d}}^{2}(\omega) d \omega .
\end{aligned}
$$

This expression shows that the disturbance signal $v(t)$ has asymptotically no effect on the parameters of the feedforward controller. On the other hand, using a white noise signal for $y_{d}(t)$ a weighted two-norm of the difference between $F$ and $P^{-1}$ is minimized using the correlation approach.

\section{SIMULATION AND EXPERIMENTAL RESULTS}

\section{A. Simulation Results}

The correlation approach for precompensator tuning is applied to a closed-loop system presented by the following transfer function.

$$
T\left(q^{-1}\right)=\frac{0.1333 q^{-1}+0.0677 q^{-2}}{1-1.5 q^{-1}+0.7 q^{-2}} .
$$

The desired output is the response of a discrete-time secondorder system given by

$$
M\left(q^{-1}\right)=\frac{0.0941 q^{-1}+0.0708 q^{-2}}{1-1.262 q^{-1}+0.4274 q^{-2}}
$$




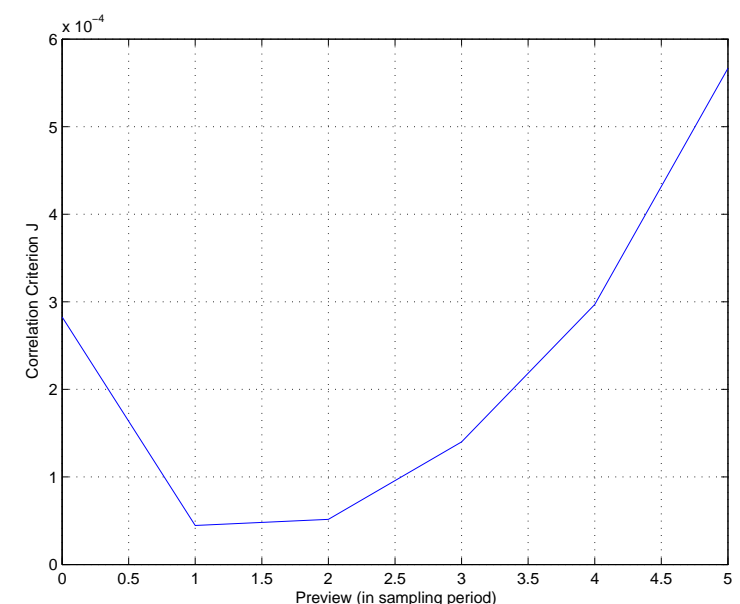

Fig. 5. Correlation criterion versus $\delta$

to a square-wave signal (between -1 and 1) of three periods (number of data is $N=600$ ). The desired output $y_{d}(t)$ is applied to the closed-loop system without precompensator to obtain the simulated measured output as:

$$
y_{m}(t)=T\left(q^{-1}\right) y_{d}(t)+S\left(q^{-1}\right) v(t)
$$

where $S\left(q^{-1}\right)=1-T\left(q^{-1}\right)$ and $v(t)$ is a uniformly distributed zero-mean white noise with a variance of 0.0225 . A non-causal controller $F\left(q^{-1}\right)=5.8253 q-8.8756+$ $4.0516 q^{-1}$ is obtained using the algorithm in Eqs. (17)-(19) with $\phi^{T}(t)=[y(t+1), y(t), y(t-1)]$ and $n=10$. It should be noted that one sampling time preview $(\delta=1)$ corresponds to the minimum of the correlation criterion when the number of controller parameters is fixed to three (see Fig. 5). Fig. 6 shows one period of the desired output $y_{d}(t)$, simulated measured output $y_{m}(t)$ and the noise-free simulated output of the closed-loop system with precompensator. It can be observed that good tracking performance can be obtained only with one set of data (the noise-free closed-loop output is almost superimposed on the desired output).

\section{B. Experimental Results}

1) System Description: The proposed precompensator tuning method is applied to a linear, permanent magnet, synchronous motor (LPMSM). LPMSM's are very stiff and have no mechanical transmission components, they, therefore, do not suffer from backlash and thus allow very high positioning accuracy to be achieved.

The motor used in the experiment is controlled by a standard two-degree-of-freedom position controller, as presented earlier, operating at a sampling frequency of $6 \mathrm{KHz}$. The motor position is measured by an analog position encoder with a period of $2 \mu \mathrm{m}$, which is interpolated to obtain a resolution of $0.24 \mathrm{~nm}$.

Experiments are carried out for both the cases of tuning a precompensator for a desired output and that of the reference model problem.

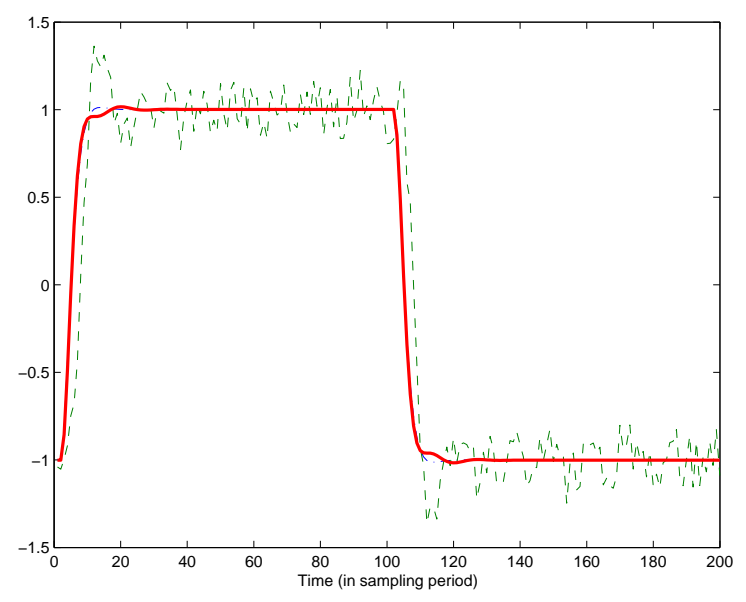

Fig. 6. measured output without precompensator (dashed), desired output (dash-dot) and noise-free simulated closed-loop output with precompensator (solid)

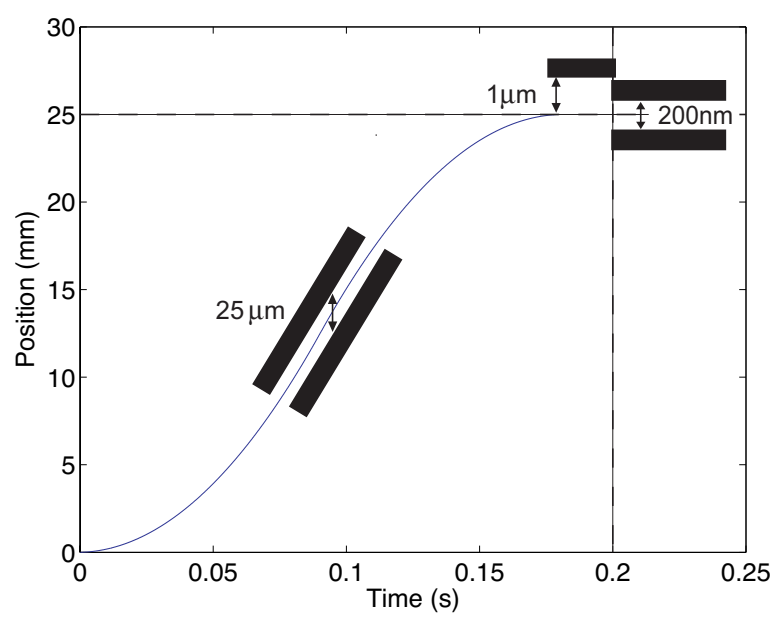

Fig. 7. Desired Output Motion

2) Tuning for a desired output: The standard movement of the motor is a so-called "S-curve", chosen to be less severe than a step motion (see Fig.7). The S-curve is defined in terms of the desired displacement, the maximum velocity, the maximum acceleration and the jerk time. It is in terms of this S-curve that the desired accuracy of the motor is described. The tracking specifications are that for an S-curve with $25 \mathrm{~mm}$ displacement, a velocity of $0.5 \mathrm{~m} / \mathrm{s}$ and an acceleration of $3 \mathrm{~m} / \mathrm{s}^{2}$, after $200 \mathrm{~ms}$ the tracking error should be less than $200 \mathrm{~nm}$ with a maximum overshoot of less than $1 \mu \mathrm{m}$. Additionally, during the transitory regime the motor position must follow the reference signal with less than a $25 \mu \mathrm{m}$ tracking error. These specifications cannot be achieved using a model-based approach because of large model uncertainties in high frequencies so a model-free approach is employed. In order to tune a precompensator for a desired output one set of data, $y_{m}$, was acquired from the system by applying the Scurve to the closed-loop system without precompensator. The 
TABLE I

THE SYSTEM TRACKING PERFORMANCE

\begin{tabular}{|c|c|c|}
\hline System & $\|e(t)\|_{\infty}$ & $\|e(t)\|_{2}$ \\
\hline \hline Without Precompensator & $0.6057 \mathrm{~mm}$ & $20.14 \mathrm{~mm}$ \\
\hline With Precompensator & $0.0014 \mathrm{~mm}$ & $0.023 \mathrm{~mm}$ \\
\hline
\end{tabular}

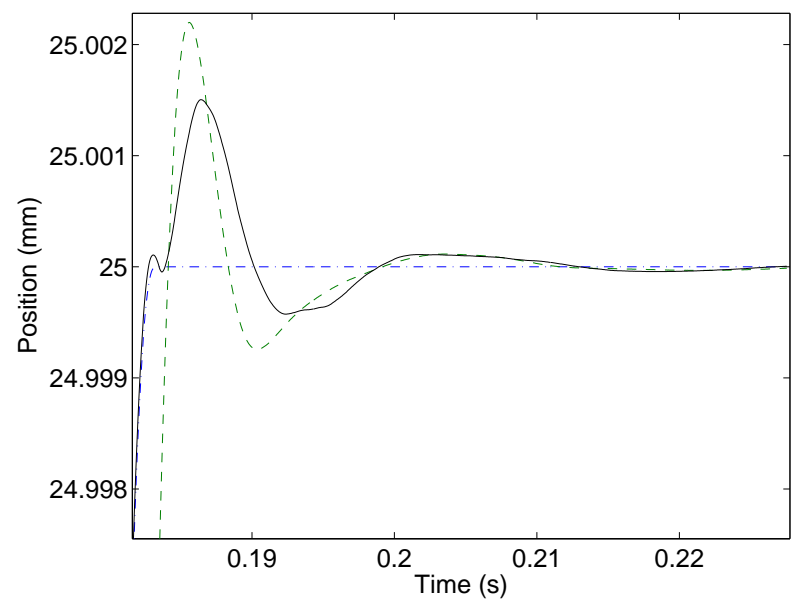

Fig. 8. Comparison of the overshoot region (Dashed : Without the precompensator, Solid : With the precompensator, Dash-dot : S-curve)

controller, $C$, was tuned previously in order to achieve robust stability. Using the proposed algorithm a precompensator $\left(n_{\rho}=3, \delta=0, n=24\right)$ was calculated and tested on the system. This precompensator meets almost all of the tracking specifications with an overshoot slightly larger than $1 \mu \mathrm{m}$ (see Fig. 8). As measures of performance the infinity norm, $\|e(t)\|_{\infty}$ and the 2-norm, $\|e(t)\|_{2}$ were taken. Table I shows the results obtained without and with the precompensator. It is clearly seen that the proposed technique greatly improves the system's tracking performance in terms of two-norm of the tracking error.

3) Tuning for a desired reference model: As remarked in Section III the proposed method can also be used to tune a precompensator for the model following problem. In this way, using an appropriate reference model, the performance in terms of overshoot can be improved. A reference model, $M$, was taken as a second order system with a natural frequency of $120 \mathrm{~Hz}$ and a damping factor of 0.9 . The $y_{d}$ used this time was a pseudo random binary signal. The proposed algorithm computes the parameters of the precompensator such that the transfer function of the closed-loop system approaches that of the reference model. Fig. 9 compares the overshoot region for the closed-loop response with and without the precompensator. This precompensator does not perform as well as the previous one specially tuned for an S-curve signal in terms of the two-norm of the tracking error, however, it gives a smaller overshoot.

\section{CONCLUSIONS}

A model-free approach to precompensator and feedforward tuning based on the correlation approach has been

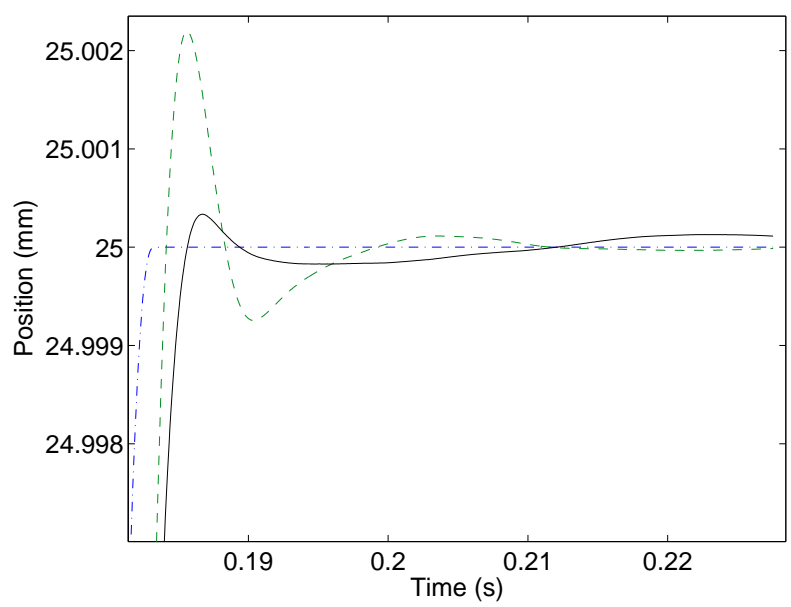

Fig. 9. Comparison of the overshoot region (Dashed : Without the precompensator, Solid : With the precompensator, Dash-dot : S-curve)

proposed. It was shown that using only one set of data and some specific tuning schemes the controller parameters can be tuned for desired output tracking or the model following problem. The approach is based on a correlation criterion which is not asymptotically sensitive to noise and can be minimized using the least squares algorithm. The frequencydomain analysis of the criterion showed that the resulting controller is a weighted approximation of the inverse of the closed-loop system (for precompensator) or the plant model (for feedforward) in the two-norm sense. The effectiveness of the method has been illustrated via simulation and experimental results.

\section{ACKNOWLEDGMENTS}

The authors acknowledge the collaborations of Ralph Coleman, Michel Mathia and Vincent Very from ETEL company for real-time experiments.

\section{REFERENCES}

[1] S. Devasia, "Should model-based inverse inputs be used as feedforward under plant uncertainty?" IEEE Transactions on Automatic Control, vol. 47, no. 11, pp. 1865-1871, 2002.

[2] H. Hjalmarsson, "Iterative feedback tuning - an overview," International Journal of Adaptive Control and Signal Processing, vol. 16, pp. 373$395,2002$.

[3] K. Hamamoto, T. Fukuda, and T. Sugie, "Iterative feedback tuning of controllers for a two-mass-spring system with friction," Control Engineering Practice, vol. 11, pp. 1061-1068, 2003.

[4] M. C. Campi, A. Lecchini, and S. M. Savaresi, "Virtual reference feedback tuning: a direct method for the design of feedback controllers," Automatica, vol. 38, pp. 1337-1346, 2002.

[5] A. Karimi, L. Mišković, and D. Bonvin, "Iterative correlation-based controller tuning: Application to a magnetic suspension system," Control Engineering Practice, vol. 11, pp. 1069-1087, 2003.

[6] L. Mišković, A. Karimi, and D. Bonvin, "Correlation-based tuning of a restricted-complexity controller for an active suspension system," European Journal of Control, vol. 9, no. 1, January 2003.

[7] A. Karimi, L. Mišković, and D. Bonvin, "Iterative correlation-based controller tuning," International Journal of Adaptive Control and Signal Processing, no. 18, pp. 645-664, 2004.

[8] D. Hoover, R. Longchamp, and J. Rosenthal, "Two-degree-of-freedom $l_{2}$-optimal tracking with preview," Automatica, vol. 40 , no. 1 , pp. $155-$ $162,2004$. 